Draft version September 17, 2018

Preprint typeset using $\mathrm{LAT}_{\mathrm{E}} \mathrm{X}$ style emulateapj v. 5/2/11

\title{
A MECHANISM FOR HYSTERESIS IN BLACK HOLE BINARY STATE TRANSITIONS
}

\author{
Mitchell C. Begelman and Philip J. Armitage \\ JILA, University of Colorado and NIST, 440 UCB, Boulder, CO 80309-0440, USA and \\ Department of Astrophysical and Planetary Sciences, University of Colorado, Boulder \\ Draft version September 17, 2018
}

\begin{abstract}
We suggest that the hysteretic cycle of black hole state transitions arises from two established properties of accretion disks: the increase in turbulent stress in disks threaded by a net magnetic field and the ability of thick (but not thin) disks to advect such a field radially. During quiescence, magnetic field loops are generated by the magnetorotational instability at the interface between the inner hot flow and outer thin disk. Vertical flux is advected into and accumulates stochastically within the inner flow, where it stimulates the turbulence so that $\alpha \sim 1$. The transition to a geometrically thin inner disk occurs when $L \sim \alpha^{2} L_{\text {Edd }} \sim L_{\text {Edd }}$, and the first "thin" disk to form is itself moderately thick, strongly magnetized, and able to advect field inward. These properties favor episodic jet production. As the accretion rate declines magnetic flux escapes, $\alpha$ decreases to $\alpha \sim 0.01-0.1$, and a hot inner flow is not re-established until $L \ll L_{\mathrm{Edd}}$. We discuss possible observational consequences of our scenario.

Subject headings: accretion, accretion disks — binaries: close — black hole physics - magnetic fields - X-rays: binaries
\end{abstract}

\section{INTRODUCTION}

Accretion onto compact objects is almost always highly variable, but in black hole binaries the variability can be particularly dramatic. Disks in these systems exhibit transitions between a luminous state in which the emission contains a substantial thermal component, and a quiescent state dominated by non-thermal emission (Remillard \& McClintock 2006). The luminous state, which can be accompanied by a non-thermal "coronal" component and disk wind (Miller et al. 2008; Ponti et al. 2012; King et al. 2013), is identified with a geometrically thin disk extending close to the innermost stable orbit (Shakura \& Sunvaev 1973), while the quiescent state is consistent with the presence of a geometrically thick hot accretion flow (Rees et al. 1982; Narayan, McClintock \& Yi 1996) and, often, a persistent jet (Fender 2001).

Hot accretion requires luminosities $L \lesssim \alpha^{2} L_{\text {Edd }}$ (where $L_{\text {Edd }}$ is the Eddington luminosity and $\alpha$ the usual viscosity parameter: Esin et al. 1997), so the existence of a transition from a thin to a thick disk as the accretion rate wanes is not surprising. More puzzling is the behavior at the start of a new outburst. Rather than backtracking in a diagram of spectral hardness versus luminosity, the system remains in a non-thermal state up to a much higher luminosity - in some cases, approaching the Eddington limit. Transient radio emission from jets is seen as the disk enters the luminous thin disk state (Fender, Belloni \& Gallo 2004). The origin of this hysteretic cycle, and much else besides, is not understood. Candidate explanations include ideas as diverse as the conjectured dependence of $\alpha$ on magnetic Prandtl number (Balbus \& Henri 2008), and instabilities associated with disk warps (Nixon \& Salvesen 2014).

In this Letter, we describe a mechanism for hysteresis based on established properties of accretion flows in

mitch@jila.colorado.edu which angular momentum is transported by the magnetorotational instability (MRI). In common with previous authors (Meier 2005; Revnolds, Garofalo \& Begelman 2006; Igumenshchev 2009; Dexter et al. 2013), we argue that dynamically significant net fields thread the inner disk while it is hot and geometrically thick. Net fields increase the strength of the MRI (Hawley et al. 1995), leading to $\alpha \sim 1$ for net fields whose ratio of gas to magnetic pressure $\beta \lesssim 10^{2}$ (Bai \& Stone 2013). With such efficient transport the density is low, and this precludes transition to a thin disk until $L \sim L_{\text {Edd }}$. Once a thin disk has formed and the accretion rate has dropped significantly below Eddington, the net flux is able to diffuse out radially through the disk (Lubow, Papaloizou \& Pringle 1994a), and $\alpha$ drops. The hot inner flow is then reestablished at a much lower $L \ll L_{\text {Edd }}$. To complete the cycle, net flux is regenerated in the inner thick disk at the interface with the adjacent thin disk. The MRI acting near the interface generates loops of vertical field, some fraction of which accumulate stochastically within the hot flow.

\section{HYSTERESIS FROM CYCLIC FLUX ACCUMULATION}

Early numerical simulations of the magnetorotational instability (Balbus \& Hawley 1998) demonstrated that although the MRI can act as a dynamo - sustaining turbulence in the absence of mean fields - the strength of MRI turbulent transport increases when a net field is present. Hawley et al. (1995), analyzing a set of unstratified local simulations that included net vertical or toroidal field, found that the saturated magnetic pressure $\left\langle B^{2} / 8 \pi\right\rangle$ and viscosity parameter $\alpha$ both scaled linearly with the net field $B_{0}$. Recent work confirms this trend. Without net field, the MRI generates an $\alpha \simeq 0.01$ (Davis et al. 2010), but this is stimulated by any net vertical field (characterized by a mid-plane ratio of gas to 


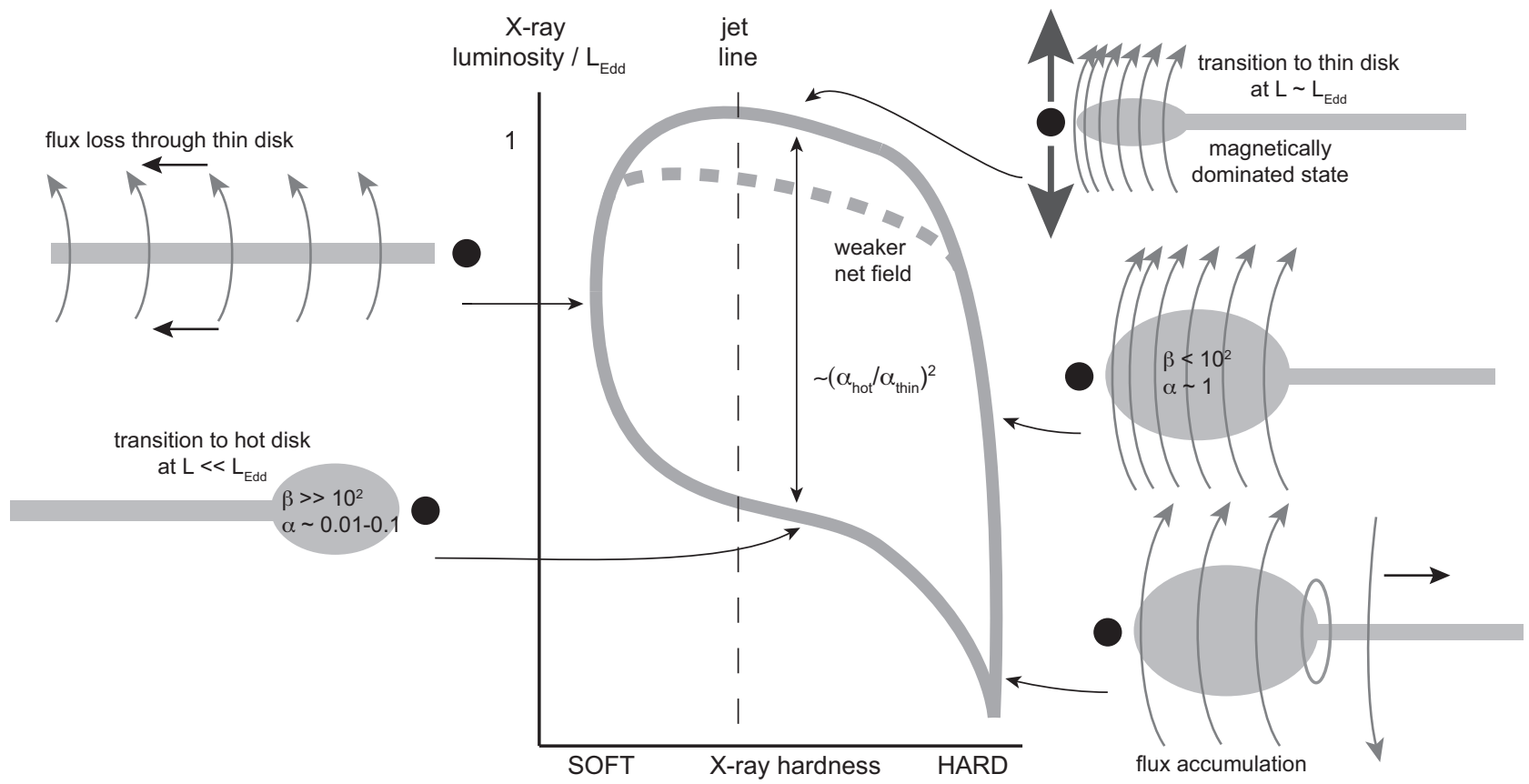

Figure 1. Illustration of the observed evolution of black hole binary disks in $L_{X}$ versus spectral hardness (simplified from Fender. Belloni \& Gallo 2004), and how that relates to the assumed evolution of net magnetic fields in our model. The luminosity of the upper branch of the transition depends on the net accumulated flux, which can vary from cycle to cycle.

magnetic pressure $\beta$ ) with

$$
\beta=\frac{\rho c_{s}^{2}}{B_{z}^{2} /(8 \pi)} \lesssim 10^{5} .
$$

Bai \& Stone (2013) find that $\alpha \simeq 0.08$ for $\beta=10^{4}$, and that $\alpha \gtrsim 1$ for $\beta=10^{2}$. Disk winds are also produced as the net field increases (Suzuki \& Inutsuka|2009; Lesur et al. 2013; Bai \& Stone 2013; Simon et al. 2013), though their strength and structure remain somewhat uncertain.

Hot accretion flows cannot exist for $L \gtrsim \alpha^{2} L_{\text {Edd }}$, because radiative cooling is too efficient (Esin et al. 1997). The hysteresis observed in black hole disk state transitions is thus consistent with the hot state possessing a substantial net flux, leading to a high $\alpha \sim 1$ and a transition luminosity approaching the Eddington limit, while the thin disk state is weakly magnetized with a lower $\alpha$ and a small transition luminosity. (The hot flow could be "magnetically dominated" with $\beta \sim 1$, as in the model of Igumenshchev (2009), but need not be provided that $\beta$ is low enough that $\alpha \sim 1$.) The simulations suggest that the gap in transition luminosities could be as large as a factor of $10^{3}$ to $10^{4}$, though less extreme values are possible if flux accumulation is inefficient or the thin disk retains some net flux. Thin disks are unable to advect vertical field inward (Lubow. Papaloizou \& Pringle 1994a), so it is plausible to expect that an initially magnetized thin disk of scale $r_{\text {out }}$ will expel its net field on the diffusion time $t_{\eta} \sim r_{\text {out }}^{2} / \eta$ associated with the turbulent diffusivity $\eta$.

If the thin disk expels net flux, how is it regenerated? We argue that flux accumulation in the vicinity of the black hole occurs due to the action of an MRI disk dynamo (King et al. 2004) at the interface between an inner hot flow and an outer thin disk. Vertical loops of magnetic field, with radial scales ranging up to $\sim h$ and zero net flux, are created at random in both the thick and thin disk regions. Loops formed deep within the inner hot flow are advected inward and do not contribute to flux accumulation, whereas loops entirely within the thin disk diffuse away. But close to the interface, field loops can be created where one footpoint is trapped in the inner hot zone while the other escapes into the thin disk. As the loop opens up and the footpoints lose causal contact, the inner hot flow is left with an element of net magnetic flux, of random sign, that is uncompensated. Inward advection of these uncorrelated elements of magnetic flux can lead to the stochastic buildup of a large net magnetic flux close to the black hole.

Because the scale height of the thin disk is so small $(h / r \sim 0.01)$, flux accumulation due to the MRI in the thin disk is probably too slow to be of interest. We therefore focus on the hot flow near the interface at radius $r$, assuming $h / r \sim 1$, a characteristic random vertical field strength corresponding to $\beta_{0}$, and a time scale for random changes in flux $t_{\mathrm{dyn}}$. The mid-plane gas pressure $P \sim \alpha^{-1}(\Omega / r) \dot{M}$, where $\dot{M}$ is the accretion rate and $\Omega$ the angular velocity. During each dynamo cycle $t_{\text {dyn }}=k \Omega^{-1}, N$ patches appear carrying positive or negative flux of magnitude

$$
|\Delta \Phi|_{N} \sim \frac{\pi r^{2}}{2 N}\left(\frac{8 \pi P}{\beta_{0}}\right)^{1 / 2}
$$

each. If a fraction $f$ of these patches escapes to the thin disk and diffuses away, a net increment of flux $(f N)^{1 / 2}|\Delta \Phi|_{N}$ is acquired by the hot flow, where it can be advected inward (Igumenshchev 2008; Beckwith et al. 2009; CaO 2011). If these flux parcels accumulate as a random walk, the hot flow will have enough net field to attain $\beta \sim 1$ after a time

$$
t \sim \frac{4 N}{f} \beta_{0} k\left(\frac{r}{r_{g}}\right)^{3 / 2} \frac{G M}{c^{3}} .
$$


Here $r_{g}=G M / c^{2}$. MRI turbulence exhibits moderately well-defined dynamo cycles, with $k \sim 10^{2}$ (e.g. O'Neill et al. 2011), and it is reasonable to assume flux cells of scale up to $\sim r$ in a thick disk. It is not clear, however, how weakly magnetized the thick disk is when it first forms, or how readily flux is separated at the interface with the thin disk. For $M=10 M_{\odot}$ and $\beta_{0}=10^{3}$ (a weak enough field that $\alpha$ would be less than unity) we have $t \sim 6 \times 10^{5}(\mathrm{~N} / \mathrm{f})\left(\mathrm{r} / 10^{3} \mathrm{r}_{\mathrm{g}}\right)^{3 / 2} \mathrm{~s}$. Provided that $\mathrm{N} / \mathrm{f}$ is of order $\sim 100$ or less this suggests that a dynamically important field can be regenerated during the quiescent phase of X-ray binaries, in far less time than the interval between outbursts. We also note that the magnetic field of the donor star will thread (at least) the outer disk, and that even if this ambient flux cannot be advected it may act as a boundary condition affecting the build up of flux within the hot flow.

Figure11illustrates how these elements may contribute to hysteresis in state transitions. In common with other authors, we assume that the action in the inner disk is sourced by large-scale variations in the accretion rate originating from further out. These variations are consistent (Coriat et al. 2012) with being due to thermal instability associated with the ionization of hydrogen (Hōshi 1979; Meyer \& Meyer-Hofmeister 1981) . For typical parameters, thermal instability originates at radii $r \sim 10^{5} r_{g}$ and can be considered independent of the physics of state transitions. The quiescent state, with a hot inner accretion flow, corresponds to the thermally unstable outer disk also being quiesent, with a low accretion rate. The extent to which magnetic flux can accumulate in the inner flow will depend on the interval between outbursts, and on the details of the flux accumulation process. Moderately strong constraints can be deduced from the observation that steady jets are present during quiescence, if one assumes that these are powered by the black hole spin (Blandford \& Znajek 1977). In this case a simple estimate for the power is $P_{B Z}=(\kappa / 4 \pi c) \Phi^{2} \Omega_{H}^{2}$, with $\Phi$ the flux threading the horizon, $\Omega_{H}$ the horizon angular velocity, and $\kappa \simeq 0.05$ a constant (for better estimates, informed by numerical simulations, see e.g. Tchekhovskov et al. 2010). The efficiency of the Blandford-Znajek process in a thick disk is then $\epsilon \propto \alpha^{-1} \beta^{-1}$, with a prefactor typically substantially below unity even for a rapidly spinning hole. Hence, even though we only require $\beta \lesssim 10^{2}$ to reach $\alpha \sim 1$, stronger fields would be needed to produce jets. A magnetically dominated accretion flow is also required in models where Quasi-Periodic Oscillations (QPOs) have frequencies linked to the magnetospheric radius (Igumenshchev 2009). These arguments are not watertight (jets, for example, could also be produced from disk winds), but they motivate consideration of models where the hot state resembles a magnetically dominated accretion flow (Dexter et al. 2013).

For $\alpha \sim 1$ the transition to a thermal state occurs at $L \sim L_{\text {Edd }}$. How this transition occurs is unclear, but if it takes place on the rapid thermal time scale the immediate consequence will be to reduce the disk pressure, so that the already strong magnetic fields become even more dominant. The high accretion rate, moreover, ensures that the nominally "thin" disk will in fact have a substantial $(h / r)$ (see e.g. the slim disk solu- tions of Abramowicz et al. 1998). These properties suggest that the newly formed inner disk will be able to advect flux inward even more efficiently than the precursor hot flow, with the angular momentum transport probably being dominated by wind loss rather than by the MRI (Lubow. Papaloizou \& Pringle 1994b; Bai \& Stone 2013). At the same time strong toroidal field will escape the disk vertically on a short time scale. The transient jets observed to form during the thick to thin transition could be a consequence of this flux expulsion (Shibata \& Uchida 1986), with an independent physical origin to the steady jets seen during quiescence.

Even after a thin disk has formed and a quasi-steady accretion state has been attained, the disk close to the black hole will still be strongly magnetized. It is likely that a corona will be present, and inflow through the magnetically dominated atmosphere of the disk will in turn affect (and slow) flux expulsion (Lovelace et al. 2009; Guilet \& Ogilvie 2012). Over time flux will escape but if initially $\beta \sim 1$ there will be a period during which $\beta$ remains $\leq 10^{2}, \alpha$ is of the order of unity, and the disk is close to the boundary between thin and hot accretion flows. Fluctuations in the accretion rate, which could originate at large scales in the disk (e.g. due to irradiation coupling between the inner disk and the thermally unstable zone: King \& Ritter 1998) or from instabilities in flows where radiation pressure is significant, will therefore be able to trigger the observed reversions of the inner disk to a hot state. Only after $\beta \geq 10^{2}$ will $\alpha$ start to drop significantly. The inner disk will then remain stably in a thin disk state until a large decline of the accretion rate has occurred. Finally the accretion rate and luminosity drop to $L \simeq \alpha^{2} L_{\mathrm{Edd}}$, with $\alpha=0.02-0.1$, closer to the value expected for a zero net-field disk. This is the level needed to re-establish a hot and initially weakly magnetized inner flow, completing the cycle.

\section{DISCUSSION}

We have argued that the hysteresis observed in the cycle of black hole X-ray binary state transitions has an elementary explanation; the hot accretion flow present in the low / hard state is more strongly magnetized than the geometrically thin disk that forms subsequently. The strength of MRI disk turbulence increases with field strength and this, in turn, results in the thick-to-thin transition occurring at a higher accretion rate than the thin-to-thick transition. The MRI can be boosted even if the disk is threaded by vertical fields that reverse sign with radius (Sorathia et al. 2010; Beckwith et al. 2011), but we have emphasized the simplest possibility that the hot flow has an organized net field. A net field can build up during quiescence, and leak away during outburst, because only thick disks (or perhaps thin disks with strong coronae) are able to advect net fields radially. The details of this flux cycle are the most uncertain aspect of our model, and numerical simulations of the evolution of magnetic fields near the interface between thick and thin disks would be valuable.

Observations indicate that the luminosity at the upper transition fluctuates considerably from source to source, and from cycle to cycle in the same source. These fluctuations might reflect the stochastic nature of flux accumulation, which could lead to a distribution of net magnetic field strengths and corresponding fluctuations 
in the value of $\alpha$. The luminosity at the lower transition, typically $\sim 10^{-2} L_{\text {Edd }}$, shows much less scatter (Maccarone 2003; Kalemci et al. 2013), suggesting that on this branch the net field is too weak to affect $\alpha$.

How and where thermal state transitions originate in black-hole binary accretion flows is uncertain, hence we are unable to propose a quantitative timeline for the cycle. However, a clear prediction of our model is that the ratio of luminosities between the cold-to-hot and hot-tocold transitions is sensitive to the ratio of $\alpha$-parameters between the highly magnetized and weakly magnetized states. Timescales that depend on $\alpha$ should reflect this dependence.

Igumenshchev (2009) and Dexter et al. (2013) have already proposed that the hot state of black hole X-ray binaries is strongly magnetized (in their case a "magnetically arrested disk": Naravan et al. 2003), and the phenomenology of jets and variability in these systems appears to be consistent with this hypothesis (Dexter et al. 2013). Igumenshchev (2009) further suggested that QPO frequencies might be linked to the magnetospheric radius of such a flow. In our model, the dynamical importance of the net field varies cyclically as the inner disk transitions from a hot flow to a thin disk and back again. This suggests observational implications beyond those described in Dexter et al. (2013). Although coronal activity is possible whenever the MRI operates in a disk, its signatures may depend on the value of $\beta$. The disk winds that are observed in absorption during the thin disk state (Miller et al. 2008; Ponti et al. 2012; King et al. 2013) may owe their prominence to being launched mainly by coronal activity (i.e., the buoyancy of turbulent fields generated by the MRI), in contrast to faster, lower-density winds launched magnetocentrifugally by the net fields expected to thread the disk prior to and immediately following the thick-to-thin transition (Suzuki \& Inutsuka 2009; Bai \& Stone 2013).

An intriguing possibility is that the collapse of the highly magnetized hot flow to a thin disk may trap an extremely strong, even suprathermal toroidal field generated by the MRI (Begelman \& Pringle 2007). The presence of such a field would thicken the disk, decreasing the viscous inflow time (Johansen \& Levin 2008), and may also lead to a large color correction in the thermal component of the spectrum, for which observational evidence may already exist (Salvesen et al. 2013; Revnolds \& Miller 2013).

We thank Jeff McClintock and Greg Salvesen for valuable discussions and comments on the manuscript. We acknowledge support from NASA's Astrophysics Theory Program under grant NNX11AE12G.

\section{REFERENCES}

Abramowicz, M. A., Czerny, B., Lasota, J. P., \& Szuszkiewicz, E. 1988, ApJ, 332, 646

Bai, X.-N., \& Stone, J. M. 2013, ApJ, 767, 30
Balbus, S. A., \& Hawley, J. F. 1998, Reviews of Modern Physics, 70, 1

Balbus, S. A., \& Henri, P. 2008, ApJ, 674, 408

Beckwith, K., Armitage, P. J., \& Simon, J. B. 2011, MNRAS, 416, 361

Beckwith, K., Hawley, J. F., \& Krolik, J. H. 2009, ApJ, 707, 428

Begelman, M. C., \& Pringle, J. E. 2007, MNRAS, 375, 1070

Blandford, R. D., \& Znajek, R. L. 1977, MNRAS, 179, 433

Cao, X. 2011, ApJ, 737, 94

Coriat, M., Fender, R. P., \& Dubus, G. 2012, MNRAS, 424, 1991

Davis, S. W., Stone, J. M., \& Pessah, M. E. 2010, ApJ, 713, 52

Dexter, J., McKinney, J. C., Markoff, S., \& Tchekhovskoy, A. 2013, MNRAS, submitted (arXiv:1312.1691)

Esin, A. A., McClintock, J. E., \& Narayan, R. 1997, ApJ, 489, 865

Fender, R. P. 2001, MNRAS, 322, 31

Fender, R. P., Belloni, T. M., \& Gallo, E. 2004, MNRAS, 355, 1105

Guilet, J., \& Ogilvie, G. I. 2012, MNRAS, 424, 2097

Hawley, J. F., Gammie, C. F., \& Balbus, S. A. 1995, ApJ, 440, 742

Hōshi, R. 1979, Prog. Theor. Phys., 61, 1307

Igumenshchev, I. V. 2008, ApJ, 677, 317

Igumenshchev, I. V. 2009, ApJ, 702, L72

Johansen, A., \& Levin, Y. 2008, A\&A, 490, 501

Kalemci, E., Dinçer, T., Tomsick, J. A., Buxton, M. M., Bailyn, C. D., \& Chun, Y. Y. 2013, ApJ, 779, id. 95

King, A. L., Miller, J. M., Raymond, J., Fabian, A. C., Reynolds, C. S., Gü ltekin, K., Cackett, E. M., Allen, S. W., Proga, D., \& Kallman, T. R. 2013, ApJ, 762, id. 103

King, A. R., Pringle, J. E., West, R. G., \& Livio, M. 2004, MNRAS, 348, 111

King, A. R., \& Ritter, H. 1998, MNRAS, 293, L42

Lesur, G., Ferreira, J., \& Ogilvie, G. I. 2013, A\&A, 550, id. A61

Lovelace, R. V. E., Rothstein, D. M., \& Bisnovatyi-Kogan, G. S. 2009, ApJ, 701, 885

Lubow, S. H., Papaloizou, J. C. B., \& Pringle, J. E. 1994a, MNRAS, 267, 235

Lubow, S. H., Papaloizou, J. C. B., \& Pringle, J. E. 1994b, MNRAS, 268, 1010

Maccarone, T. J. 2003, A\&A, 409, 697

Meier, D. 2005, in From X-Ray Binaries to Quasars: Black Hole Accretion on All Mass Scales, ed. T. J. Maccarone, R. P. Fender, \& L. C. Ho (Dordrecht: Kluwer), 55

Meyer, F., \& Meyer-Hofmeister, E. 1981, A\&A, 104, L10

Miller, J. M., Raymond, J., Reynolds, C. S., Fabian, A. C., Kallman, T. R., \& Homan, J. 2008, ApJ, 680, 1359

Narayan, R., Igumenshchev, I. V., \& Abramowicz, M. A. 2003, PASJ, 55, L69

Narayan, R., McClintock, J. E., \& Yi, Insu 1996, ApJ, 457, 821

Nixon, C., \& Salvesen, G. 2014, MNRAS, 437, 3994

O'Neill, S. M., Reynolds, C. S., Miller, M. C., \& Sorathia, K. A. 2011

Ponti, G., Fender, R. P., Begelman, M. C., Dunn, R. J. H., Neilsen, J., \& Coriat, M. 2012, MNRAS, 422, L11

Rees, M. J., Begelman, M. C., Blandford, R. D., \& Phinney, E. S. 1982, Nature, 295, 17

Remillard, R. A., \& McClintock, J. E. 2006, ARA\&A, 44, 49

Reynolds, C. S., Garofalo, D., \& Begelman, M. C. 2006, ApJ, 651,1023

Reynolds, M. T., \& Miller, J. M. 2013, ApJ, 769, id. 16

Salvesen, G., Miller, J. M., Reis, R. C., \& Begelman, M. C. 2013 , MNRAS, 431, 3510

Shakura, N. I., \& Sunyaev, R. A. 1973, A\&A, 24, 337

Shibata, K., \& Uchida, Y. 1986, PASJ, 38, 631

Simon, J. B., Bai, X., Armitage, P. J., Stone, J. M., \& Beckwith, K. 2013, ApJ, 775, article id. 73

Sorathia, K. A., Reynolds, C. S., \& Armitage, P. J. 2010, ApJ, 712,1241

Suzuki, T. K., \& Inutsuka, S. 2009, ApJ, 691, L49

Tchekhovskoy, A., Narayan, R., \& McKinney, J. C. 2010, ApJ, 711,50 